Final submitted and accepted version

Reichenberger, I. (2017). Digital nomads - a quest for holistic freedom in work and leisure. Annals of Leisure Research, 21(3), 364-380. 


\title{
Digital Nomads - A Quest for Holistic Freedom in Work and Leisure
}

\begin{abstract}
Digital nomads are portrayed as young professionals working solely in an online environment while leading a location independent and often travel reliant lifestyle where the boundaries between work, leisure and travel appear blurred. This paper aims to conceptualize the digital nomad phenomenon by establishing a definition of digital nomads. Further, it explores their motivations for adapting this lifestyle and how these are addressed in practice, and examines how work, leisure and travel are interpreted. Digital nomads aim to create a holistic lifestyle characterized by comprehensive freedom where both areas of life are regarded as equally enjoyable and do so through professional, spatial and personal freedom. Ideally, digital nomads perceive work not as an imposed obligation but regard it - much as their leisure activities - as intrinsically motivated and fulfilling. Although crucial for a positive perception of this lifestyle, travel comes with personal challenges that are considered a different type of work.
\end{abstract}

\section{Keywords}

digital nomads; leisure; work; lifestyle; location independence; holism 


\section{Introduction}

The role of information and communication technologies for tourism has long been a popular topic of academic research (e.g. Ali \& Frew, 2014; Berne, Garcia-Gonzalez, \& Mugica, 2012; Buhalis \& O'Connor, 2005; Werthner \& Klein, 1999). Journalistic sources however suggest that ICT impacts extend beyond the tourism supply perspective, perhaps changing the potential role of travel within life. In particular, this coverage refers to individuals selfidentifying as 'digital nomads'. These appear to be young professionals whose ICT-based occupation allows them to work location independently - an advantage heavily used through long, frequent and sometimes constant travels while simultaneously earning the required income (e.g. Lamarque, 2015; Makimoto \& Manners, 1997; Spinks, 2015). Media coverage and blog articles indicate that the main driver behind adopting this travel-based lifestyle is a desire to escape inhibiting structures of a traditional, location-dependent working existence. This is considered to leave little time for leisure, often combined with a perceived alienation or disconnect from work-related tasks (e.g. Altringer, 2015; Snedden, 2013). Instead, digital nomads appear to strive for a more holistic approach to life where work and leisure are not considered dichotomous through spatial and temporal separation, but where both aspects of life contribute equally to self-actualization, -development and -fulfilment (Biesalski, 2016, n.d.; Blanda, 2016; David, 2014).

The academic literature has not yet acknowledged this phenomenon. Even Dal Fiore, Mokhtarian, Salomon, and Singer (2014), when examining the impact of new technologies on travel behaviour, were unable to identify a definition other than Wikipedia's 'Digital nomads are individuals who use telecommunications technologies to earn a living and, more generally, conduct their life in a nomadic manner. Such workers typically work remotelyfrom home, coffee shops, public libraries, and even recreational vehicles- to accomplish tasks and goals that traditionally took place in a single, stationary workplace' (Wikipedia, 2016). Current knowledge is thus limited by its potentially biased and selective origin (e.g. newspaper and online articles, interviews, blogs). No reliable information is available to shed light on a lifestyle that, considering increased flexibility in working arrangements and relevance of ICTs, can be expected to become more common (a google.com search for 'digital nomad' generated 804,000 results in February 2017). This paper contributes to a better understanding of this yet unexplored type of traveller, and provides insight into how the frequently discussed work-leisure-relationship may be changing due to societal and 
technological changes. It aims to explore digital nomads with the following objectives. First, it is necessary to establish an academic definition of digital nomads - understanding the main characteristics that 'make' a digital nomad will provide a conceptualization for determining the focus of both this and future research. Second, the motivations for adapting a digital nomad lifestyle and how they are addressed in practice will be examined. This will further the understanding of what digital nomads expect from travel, leisure and work and how these components are interrelated. Third, in light of seemingly blurred boundaries between work and leisure through dominant travel, perceptions of work and leisure are to be discussed. This contributes to the often controversial discussion on the interdependencies between different areas of life. This research thus aims to answer the following research questions: 'What is a digital nomad?', 'How does this lifestyle address the motivations of digital nomads?', and 'How do digital nomads interpret work, leisure and travel?' Existing literature will first be reviewed to discuss the relationship between and purpose of leisure and work, taking into account alternative approaches to life where both realms are non-dichotomous.

\section{The Leisure-Work-Relationship}

Although discussed for decades, there is still no common agreement on what is work and what is leisure and how both are connected. Due to technological, economic and social changes (Haworth \& Veal, 2004), it is 'necessary to ask repeatedly whether we need to revise our notions about what leisure is' (Roberts, 2011, p. 5). Leisure as work's counterpart was originally considered a secondary, dependent variable (Hilbrecht, 2007); based on the often communicated desire of digital nomads to increase leisure (e.g. Russell, 2013; Walsh, 2012) this paper places the concept of leisure in the centre of the discussion and explores the role of work in a second step and in relation to it.

Early attempts to define leisure were founded simply upon the absence of work, thus lacking 'an intrinsic character of its own' (Beatty \& Torbert, 2003, p. 240). Further explorations considered leisure time as an available quantity after employment-related commitments were concluded (Robinson \& Godbey, 1997), neglecting everyday tasks and errands. Thus, more comprehensive approaches emerged to include behavioural aspects of leisure, assigning only certain activities to the leisure realm (Beatty \& Torbert, 2003; Robinson \& Godbey, 1997) assuming that individuals experience these activities as enjoyable and neglecting the notion 
that leisure is predominantly an activity of the mind (de Grazia, 1962, in Bowers, 2011). An expansion of the concept through experiential aspects thus based leisure on individual perceptions as opposed to external parameters. Some components of perceived leisure may be enjoyment, relaxation, a lack of evaluation, and an underlying intrinsic motivation (Shaw, 2009), but especially freedom (of choice) has emerged throughout numerous studies as a prerequisite (Kleiber, 2009; Mokhtarian, Salomon, \& Handy, 2006; Moore, Cushman, \& Simmons, 1995; Shaw, 2009). All components however remain subjective: The individual framework is what determines - on a case-by-case basis - whether certain activities or behaviours are to be classified as leisurely (Beatty \& Torbert, 2003; Hamilton-Smith, 2006; Hilbrecht, 2007).

Based on these characteristics, perceived leisure must not necessarily be confined to situations outside of paid employment or required tasks and errands. The perception of and assignment to either realm depends - in addition to the prior components - on whether or not a sense of flow is evoked (Primeau, 1996), where individuals are in a state of full absorption while skills and challenge are perfectly balanced (Csikszentmihalyi, 1991). This increases the difficulty of distinguishing between work and leisure - if such a thing is indeed possible -, especially since current research tends to focus on a perceived lack of leisure as work appears to become more dominant (Haworth \& Lewis, 2005; Haworth \& Veal, 2004). This is in part due to increasingly blurred lines between what is work and what is free time (Lobo, 2006), induced by non-standardized work hours, home office arrangements, accessibility through ICTs and the rise of flexible contracts (Breedveld, 1996; Lobo, 2006; Reichenberger \& Schäfer, 2016). These merging boundaries make free time not only less available but also more fragmented and challenging to achieve, although there is no doubt about the benefits of recuperation from work through leisure activities in their contribution to well-being (Cropley \& Millward, 2009; Haworth \& Veal, 2004; Roberts, 2011).

Taking into account the relevance of perception as determined by individual frameworks and flow, this may only then become an issue when what is traditionally considered work does not adhere to leisure-related criteria and both realms fulfil profoundly different roles in life. Parker (1971) describes this state as segmentalism, whereas in a holistic relationship work and leisure are equally integrated. The context of the so-called 'New Economy' (Kelly, 1998) encourages this holistic relationship, as fewer boundaries between the two concepts based on technological and societal changes may lead to greater fulfilment and meaning in work 
(Lewis, 2003), which is subsequently considered the new leisure. However, especially the factor freedom is often difficult to achieve in employment-related environments. In order to truly experience leisure within work, hierarchy and domination must be eliminated (Bookchin, 1982), as must be evaluation - the nature of work must thus be changed to allow for freedom and intrinsic self-motivation (Stormann, 1989). Otherwise holism cannot be achieved, work remains without personal meaning, and a sense of alienation or detachment may occur. This is especially challenging as both work and leisure are crucial in the creation of self-identity and personal worth (Best, 2010; Reid \& Mannell, 1994; Roberts, 2011; Stormann, 1989) - if work relies on extrinsic motivation, combined with low levels of freedom and control, this purpose is difficult to fulfil. But finding sufficient opportunity for balance through leisure becomes increasingly difficult in performance-oriented consumerist societies (Bowers, 2011). In addition, leisure can suffer from spillover effects where negatively perceived work negatively impacts the fulfilment gained within free time (Surber, 1983). One coping mechanism that has emerged is the so-called downshifting (Hilbrecht, 2007), in which working hours and income are reduced in a conscious attempt to transfer the focus from materialism to leading more fulfilling and balanced lives (Hamilton \& Mail, 2003). Bowers (2011) identified similar trends in a study examining new approaches to the work-leisure-relationship, where individuals attempted to reframe lifestyles to live a selfcontrolled, meaningful and more seamless life by extracting themselves from societal expectations and the traditional temporal work obligations.

These approaches appear to exhibit similarities with the aims of digital nomads, who seem to seek to eliminate dissatisfaction with structures perceived as inhibiting freedom, a lacking work-life-balance and a perceived disconnect/alienation through a lifestyle that makes travel not an occasional and temporally restricted leisure activity but a prominent, if not constant, part of life. For lack of more suitable expressions, unless explicitly stated otherwise, this paper will continue to use the term 'work' to refer to activities for paid employment and 'leisure' to refer to activities that are either conducted during free time or fulfil the previously discussed potential criteria of freedom of choice, intrinsic motivation, enjoyment, relaxation and/or lack of evaluation.

Before discussing digital nomad characteristics, whether their motivations actually stem from the challenges as illustrated in the theoretical discussion, and how work, leisure and travel are interpreted within their lives, the methodology of the study will be discussed. 


\section{Methods}

Due to the exploratory nature of this study, the parameters required for a quantifiable characterization of the phenomenon are yet to be identified. The fragmented and nonscientific nature of information available on digital nomads, the lack of agreement on the concepts of leisure and work, and the role of subjective perception of what is work and leisure call for a qualitative approach based upon social constructivism. Social constructivism centres around the lived experience, acknowledging its subjectivity in that reality is a self-created mental construct built through experiences (Small, 1999). The focus is thus to understand individual interpretations and perceptions of reality (Lincoln, Lynham, \& Guba, 2011) by exploring "the meaning-making activity of individuals" (Pernecky, 2012, p. 1132). Through this, individual motivations and subjective interpretations of traditionally separated areas of life can be uncovered. To answer the research questions as illustrated prior, data was collected using both content analysis of online material to draw upon existing non-academic information, and semi-structured in-depth interviews to confirm and further explore onlinebased information through the cohesive narrative of individual experiences - both methods linked to and suitable for interpretive constructivist approaches (Angen, 2000).

In a first step, an online content analysis was conducted. Online-based information from, for and about digital nomads (e.g. interviews, articles, reports, videos, personal blogs, forums, discussion pages) was identified through an initial Google search for 'digital nomads' and subsequent links or references to further websites. Due to the clearly stated research aims, a directed approach was used to focus specifically on evidence relevant to the research questions (Hsieh \& Shannon, 2005). Particular attention was thus paid to digital nomad characteristics/identifiers, motivations for adapting the lifestyle, the lived reality of digital nomadism, and work, leisure and travel perceptions and behaviour. This information was subsequently imported to NVivo, where contents were first assigned to each of the three research questions to create a first structure within the extensive data, resulting in three individual yet interconnected document collections. In a second step, each collection was analysed inductively to create emerging codes according to similarities in perceptions and experiences. A third step examined potential interdependencies and overlaps between collections and their respective codes to ensure a comprehensive foundation for the second data collection step. 
This step consisted of 22 semi-structured in-depth interviews, further exploring why and how this lifestyle is conducted and supplementing explanatory detail to understand not only the 'what' but also the 'why' through cohesive narratives. As no definition of digital nomads exists, it was not known what criteria the subjects of this study should adhere to. Due to the limitations in available information, and the aim of the research to provide a definition of digital nomads while exploring the interpretation of different areas of life, self-identification was chosen as the criteria to select potential participants. Every individual identifying as a digital nomad was thus considered part of the population, so as not to prematurely eliminate potential participants who do not conform to the unconfirmed data stemming from the content analysis. This approach is subject to self-selection bias, as it is likely that the majority of the sample holds particularly strong preferences for the topic (Li \& Hitt, 2008), in turn leading to a potential over-reliance on extreme or critical cases. However, these have been found to be particularly helpful when the research aim is not confirmation but discovery (Seawright, 2016), as they "yield the most information and have the greatest impact on the development of knowledge" (Patton, 2015, p. 276). Participants were recruited through Facebook postings in three different international English speaking digital nomad groups ${ }^{1}$. Groups were selected based on the number of members and continuous activity as evidenced by several postings per day. Interviews were then conducted via Skype with every individual willing to participate and considering themselves a digital nomad. The social constructivist researcher is regarded as an active co-creator of knowledge throughout the data collection process, thus having significant control over the way in which and the type of knowledge that emerges (Lincoln et al., 2011). To reduce this potential impact, interviews did not follow a clear question sequence. Instead, participants were informed about the purpose of the research and encouraged to initially provide self-selected information on their lived reality as a digital nomad and its underlying motivations. Through this, it was not the researcher who determined which aspects of their motivations, perceptions and practice were relevant for understanding digital nomads, but the individuals themselves. The interviewer then ensured to draw out further information to elaborate on the critical components of the content analysis, paying particular attention to digital nomad characteristics, the different underlying motivations and how these are addressed in practice, and the individual perceptions and experiences of the components work, leisure and travel. The subsequent analysis and coding was then conducted twice - the first coding step took into account only the interview material to identify their

${ }^{1}$ https://www.facebook.com/groups/1428340887415620/

https://www.facebook.com/groups/901887786539278/

https://www.facebook.com/groups/756306204446638/ 
independent themes and structures based upon the original three research questions. The second step compared the results of the first coding stage with the content analysis data to create one cohesive and comprehensive data set.

Table 1 provides an overview of the participants of the study including their age, nationality and professional occupations. Four out of the 22 participants reported to travel/live with a partner, with the remaining individuals being single. It must be noted that all participants willingly chose this particular lifestyle, as opposed to being forced due to not being able to find 'traditional' location-dependent employment.

\section{Insert Table 1 here}

\section{Results}

Results are presented in the following sections, beginning with a definitional approach and an overview of the motivations underlying this particular lifestyle. Subsequently, work and leisure are discussed, exploring how, in what form, and to what purpose these are integrated in a digital nomad lifestyle. Although travel is traditionally considered a form of leisure, in this particular case it is discussed separately due to the overarching importance of mobility.

\section{Digital Nomad Definition}

Media reports (e.g. Bennion, 2013; Hart, 2015; Investopedia, 2017) as well as the content analysis portray digital nomads as predominantly young professionals who live what they refer to as a location independent lifestyle by being able to conduct their work in an online environment, simultaneously working and travelling internationally. However, a more critical and heterogeneous approach to digital nomads emerged throughout the interviews to address the first research question 'What is a digital nomad?'.

The youngest participant was 19, whereas the oldest participant was 51 years old. The majority of participants were in their 20s and early 30s, perhaps due to the absence of family 
commitments at earlier stages in life as well as the fact that this generation developed an affinity to ICTs early in life, thus reducing entry barriers and perceived obstructions. Nearly all participants completed at least an undergraduate degree and were currently working as either freelancers, entrepreneurs, or a combination of both, with only one remote employee represented in the sample. Greater distinctions emerged when looking at the role of international travel, often emphasized to highlight the advantages of a digital nomad lifestyle. Although most participants either have no permanent residence or only spend up to three months a year within their usual 'home' environment, they did not consider the extent of travel as a criterion for being a digital nomad. Instead, mobility and travel were regarded as two connected, yet independent features of what makes a digital nomad, stating that a digital nomad may also utilize location independence only to be mobile within a comparatively restricted geographical space such as one's home environment.

The digital nomad lifestyle is about having the freedom to work anywhere. There's the full-time digital nomad, those that are without a permanent residence for years, then there are the part-time digital nomads that have a homebase and travel for several months. And then there are those who mostly live at home, those who have the opportunity to take their business with them for a couple of weeks or simply work in different coffee shops or co-working spaces, who just have that option but don't make travel a part of their everyday life.

Female, Germany, 33

For me, the transitions are very fluent. Of course you have the extreme ones, those with no permanent residence at all, they're mostly younger and don't even know a life without internet. But we are the older generation, giving it a try, taking some time off to see what this is like. A lot of people may disagree with me, many connect being a digital nomad to international travel, but why not just go to a coffee shop? Or work underneath a tree in the park? As long as you can pick your location, and actually do it, you're a digital nomad.

Female, Germany, 45 
For me, a digital nomad is anyone who is location independent and actually uses it. People use it in different ways, I have met many, many digital nomads through my job, and there's everything from taking a few weeks every year to travelling without interruption for years. (...) But I guess technically, you don't need to travel at all, you can just go to a café in your city, as long as you move.

Male, Argentina, 31

Similar distinctions emerged from other participants, emphasizing the utilization of location independence through online-based work by translating it into location mobility and its different extents. Demographic variables or other factors were not presented when elaborating on digital nomad characteristics, however a hierarchy of digital nomadism based on the commitment to travel was described by all. The definitional approach in Table 2 highlights those levels by regarding the opportunity for location independence as a precondition. Travel (domestic as well as international) is thus not regarded as a necessary requirement for being a digital nomad but rather one possible way of utilizing location mobility. While Level 0 only describes the basic requirements for becoming a digital nomad (namely location independence through online-based work), individuals within Level 1 enter the digital nomad realm through somewhat restricted mobility. Level 1 digital nomads may thus be those individuals that, as described in the quotes above, utilize their location independence on a limited spatial level while continuously remaining within their home environment. Level 2 then includes occasional and intermittent travel with subsequent returns to the home environment termed 'homebase'. In these cases, a permanent residence is maintained and travel is limited to restricted periods of time. Level 3 is characterized by constant full-time travel with no permanent residence or homebase to return to, thus maximizing the location independence provided by their working conditions.

\section{Insert Table 2 here}

All members of the sample are classified as digital nomads on the second or third level, perhaps due to self-identification as a digital nomad being the sampling criteria. First level digital nomads may not necessarily consider themselves as such due to the heavily emphasized role of travelling in common depictions of this terminology. In addition, first level digital nomads are of limited interest for this study, as the role of travel and its relation to work and leisure is to be explored in later sections of the paper. 


\section{Motivations}

To answer the second research question, 'How does this lifestyle address the motivations of digital nomads?', common motivations and their manifestation in practice are now to be examined. One of the possible motivations behind adapting a digital nomad lifestyle, the desire to escape the structures of a traditional location dependent working style, was already touched upon in the introduction and emerged as one of the main motivators throughout the online content analysis and subsequent interviews. All participants used the term 'freedom' to illustrate their individual reasons behind adapting a location independent lifestyle through online-based work, thus utilizing the expression that also emerged throughout the theoretical discussion as a prerequisite for intrinsically motivated, fulfilling and enjoyable activities - in the realms of both work and leisure. Freedom then appeared in a variety of contexts, including freedom within paid employment, freedom relating to location independence and freedom to pursue self-development.

Professional freedom referred to the motivation to select and structure work related tasks in a self-imposed manner. Often driven by the desire to create a more flexible and tailored life outside of externally imposed structures (e.g. specific working times, restricted free time, geographical dependence), building (or in the case of freelancers, choosing) one's own business allows participants to have autonomy over not only the circumstances within which they work, but also greater control over work itself. Focusing on projects, services or tasks that participants were personally passionate about, in a way that they had full control over, led to an increased feeling of connectedness and purpose - for 16 out of the 22 participants, this was to address a sense of alienation they had experienced in prior paid employment.

I had a normal job in marketing before, and I got sick of it very quickly. I was working for someone else's dream, helping others to do what they love and to do it well, but what they loved wasn't what I loved, who was doing it for me? I'm the only one who can do that, and I didn't get the satisfaction out of it because it wasn't for me, it wasn't what I value.

Female, Ireland, 27 
The digital nomads represented in the sample thus consciously aim to eliminate hierarchy, domination (Bookchin, 1982) and external evaluation (Shaw, 2009), creating the basic requirements for work being able to fulfil leisurely purposes in that it aids the creation of identity, self-worth, self-development, recuperation and enjoyment. This professional freedom must be designed in a way that it can be executed through online channels only in order to achieve location independence.

Location independence in the form of domestic and international travel emerged as spatial freedom, highlighting the motivation to live and work in a variety of places and inextricably connected with freedom to learn and experience. Exposure to different cultures, ways of life, values, norms, lifestyles and opinions that can be accessed through travel was considered an especially important motivation to achieve spatial freedom. Due to the ability to work anywhere, these opportunities are not restricted to weekends or vacations but can be extended to longer periods of time.

For me, when I travel I want to immerse in the new culture (...), being a nomad is being immersed in a new environment. For me it's really exploring, try to live in a way I really like. I don't think capitalist systems where we are make people happy, it's finding a sustainable lifestyle that puts the person at the centre and not the growth of the economy. And the more I live in other places, other cultures, really get to know other people, the more I am finding my place.

Male, Italy, 44

Spatial freedom and the exposure to the new and different was often argued to stem from a dissatisfaction with the systems of the original home environment, re-emphasizing the motivations behind downshifting (Hamilton \& Mail, 2003) and transferring value from materialist/consumption oriented approaches to individual self-centred fulfilment. Another effect of this spatial freedom and the subsequent learning experiences is creativity. Onlinebased entrepreneurs or freelancers (as opposed to remote employees with a lower level of professional freedom) consistently emphasized the value of changing circumstances and environments on their creativity and thus business success, positively impacting their professional freedom. Simultaneously, the flexibility to select locations with lower costs of living allowed them to develop their business with less financial pressure. 
The autonomy over both spatial movements and professional activities then resulted in personal freedom, beneficial for productivity, creativity, and most importantly selfdevelopment. The previously addressed focus on material goods or financial acquisitions, often considered superficial and not contributing to participants' happiness, has been replaced by a motivation to further skills, knowledge and self-improvement - on both a professional and personal level. This self-development is enabled and enhanced by exposure to other cultures and ways of life as illustrated through spatial freedom, the challenges of being solely responsible for one's business and income without the securities attached to permanent contracts and regular salaries, or the opportunities to learn languages or other skills in suitable environments.

In summary, professional, spatial and personal freedom are inextricably connected and one cannot be fulfilled without the others (see Graph 1). Only when professional freedom in terms of autonomy is achieved in combination with the utilization of spatial freedom can personal freedom be developed within the desired parameters, and only then can the desired outcome of digital nomad holism be achieved. Graph 1 highlights the strong connection between work (professional freedom) and leisure (personal freedom), as freedom in both areas contributes to learning, the acquisition or advancement of skills, and self-development. Location independence (spatial freedom) increases leisure (personal freedom) through experiences and exposures through travel, while also contributing positively to the perceived control over work (professional freedom) through movement and the stimulation of creativity. If all motivations are fulfilled, digital nomads are able to achieve the holistic state that they are seeking.

\section{Insert Graph 1 here}

\section{Leisure and Work in the context of Travel}

The prior discussion already suggests that digital nomads aim for professional freedom in order to transfer selected leisurely components (such as enjoyment, self-control) to their working environment - yet when prompted to evaluate how professional and personal freedom are manifested in everyday life, participants still adhered to the traditional dichotomous terminology of work versus leisure/free time. This distinction, however, appeared to be utilized predominantly for structuring time to ensure sufficient monetary 
compensation without differentiating based on perception of tasks. To answer the research question 'How do digital nomads interpret work, leisure and travel?', it is now examined how work-related activities are perceived, if participants achieved a transfer to the leisure realm, and what role travel - traditionally considered a leisure activity - plays when it is not the exception but the norm.

Especially entrepreneurs, whose business corresponds with individual interests, skills and passions, perceived employment related tasks as predominantly positive: fulfilling, enjoyable and both intrinsically and extrinsically motivated. Those that had already established a successful business highlighted the positive component of being able to switch between work and leisure based on situational preferences and current abilities in terms of time, focus and creativity. This freedom, combined with an autonomous selection of what participants were working on, frequently replaced the notion of having to do something with being able to do something.

You know the saying 'If you find work that you love, you'll never work a day in your life' or something? So cheesy, but also true. The work I do and how I can do it, I enjoy it so much that it doesn't feel like work. (...) It means, I guess what bothered me before was that I didn't like all aspects of my job, some were boring, some I wasn't really good at, but I had to, even if I didn't have the mindset at the time, on someone else's terms. So now, there still is a clear line between work and play, because, you know, money, but it doesn't really feel that different to me anymore.

Male, Netherlands, 31

Nevertheless, added pressure especially regarding their financial situation was an issue raised by the majority of participants that were in the early stages of business development. In these cases, the autonomy that allowed established digital nomads to enhance their free time and creativity was not necessarily utilized for this purpose, as professional freedom was considered the prerequisite for being able to maintain spatial and personal freedom and thus took predominance.

I guess at the start I was more panicking, like oh, we'll never make it work and I was nervous about it. And to be honest, I kind of got a bit resentful, I was in this beautiful place with so much to do, and was stuck with the laptop and couldn't enjoy it, and I was like, you know, that's not how this was supposed to be. So nothing was fun, work 
wasn't fun because of the pressure, and everything else wasn't fun anymore because I felt bad about not working. But now the money is coming in, regularly, and I'm learning to find my balance.

Female, Ireland, 25

Similar themes emerged throughout other interviews - when the extrinsic motivation of work became more dominant than the intrinsic motivation, when spatial and personal freedom were perceived as jeopardized, or when too little balance between activities existed, even otherwise fulfilling employment tasks were not perceived as leisurely, in turn confirming the applicability of the spillover effect (Surber, 1983) to participants in both directions. Equally, the positive perception of traditional leisure activities was often reported to decrease if work related tasks were subsiding.

I realized that the four hour work week sucks as well, because I don't feel like I'm contributing anything to the world and you feel like you don't have a goal and a purpose. It's not just about going surfing, I really want to help people live their dream, this is how I live my dream. So during those times where I didn't work much, I realized that I'm not enjoying what else I'm doing very much, there was no balance.

Female, Germany, 33

The frequent use of the term 'balance' highlights the importance of the holistic lifestyle where both work and leisure contribute equally to self-identity and a feeling of self-worth. If professional and personal freedom are utilized to their advantage and are predominantly intrinsically motivated, participants reported to be at their happiest and most fulfilled. Deviations from perceived balance led to dissatisfaction and a decrease in enjoyment of any activity. Although work and leisure/free time/play was commonly used terminology, when looking closer at the perceptions of activities assigned to either realm, no difference is apparent. It must however be noted that all participants reported to be very satisfied with their current situation, which is unlikely to be applicable to all digital nomads. The perspectives and experiences of those who have not achieved professional freedom to the required extent are not represented in this study.

Most of the previous discussion on the perception of work and leisure in a digital nomad context is likely to also be applicable to other self-employed individuals, as many of the 
advantages can be traced back to greater control over the structure and utilization of time (Blanchflower, 2004; Buttner \& Moore, 1997). Although digital nomadism enables living in destinations with lower costs of living, thus reducing financial pressure, the role of spatial freedom becomes more apparent when examining type and perception of non-employment related activities whose existence and purpose often stems from travel.

Activities named by participants encompassed obligations, social, self-development and tourism. Obligations referred to day-to-day requirements (e.g. cleaning, grocery shopping) as well as acquiring competences on how to manage everyday life in new destinations where structures, systems and culture were often unfamiliar. These tasks were frequently considered stressful, frustrating and challenging, due to the repeated effort and persistence required to navigate them successfully while being predominantly extrinsically motivated. Spatial freedom thus included several negatively perceived components, especially for third level digital nomads who moved between destinations regularly and were forced to create a stable environment due to their length of stay (usually two to three months) and the absence of such in form of a homebase.

I love exploring new places, but getting established in them, that's something I still don't enjoy. Find a place to live, find a place to work, find good restaurants and cafes, find friends, figure out how things work, the red tape, the culture that's different. It's like having a culture shock every few months. New nomads don't see that. You spend days and days getting nowhere, knowing no one, it's lonely, and then if your business isn't good yet, it can be bad.

Male, Greece, 28

These obligations did little to enhance well-being, often had the opposite effect and were thus not perceived to fall within the leisure realm. Similarly, social activities were sometimes considered strenuous especially for participants who described themselves as more introverted and implicated an absence of the state of flow in social situations. The large majority of participants led this lifestyle without the presence of a partner or friend, in turn emphasizingthe importance of social networks.

It can be very, very lonely. You don't have your friends and your family, and have to deal with a lot of challenges, in life and in work. So you need to find new friends where you are, and for some people that's easy and fun, but for me it's actually hard 
work because it doesn't come naturally, the social stuff. Putting yourself out there, going to co-working spaces, to meet ups, and then they leave or you leave and it starts again.

Female, Sweden, 26

While obligations and some social activities were not necessarily perceived as leisurely, activities relating to self-development and tourism were. Tourism-related activities included sightseeing and the mostly independent exploration of the destination while getting to know the local culture. Self-development activities then differed strongly individually, but mostly referred to acquiring or furthering skills in hobbies (e.g. arts, sports) as well as spiritual and intrinsic self-development (e.g. retreats, yoga, health, meditation). Activities in both areas were freely chosen based solely upon an intrinsic motivation to address the advantages as discussed in the sections on spatial and personal freedom and could, as such, not be conducted to the same effect in the participants' traditional home environment.

When you travel, you're not leaving yourself behind, your problems always come with you. (...) It gives me the opportunity to work this out, to find myself, to find out what I want, what makes me happy, to become a better me, and travel plays a huge role. (...) The touristy things are only part of it, where you see what else is out there, you get new inputs, it's exciting and fun, and it also makes me much more creative at work. (...) But I also have the freedom to do and learn, I can do what I want, and if I want to learn how to surf I can go to Bali, and if I want to do a yoga retreat, I can do it in Thailand: Having all these options, this is why I do this.

Female, Germany, 35

In summary, it can be said that if professional, spatial and personal freedom can be utilized to their best advantage, participants did, in fact, perceive employment-related work as leisure while also retaining the leisure aspect of many activities undertaken in their free time. Although travel is a large component of the three types of motivational freedoms being fulfilled, it is not exclusively perceived as a positive experience. Certain aspects that come with a location independent lifestyle are regarded as challenging, often referred to as 'work' in the sense that they are non-enjoyable, often difficult to accomplish, yet necessary. While the holistic lifestyle of digital nomads aims to eliminate extrinsically motivated activities that do not evoke a sense of flow, these appear to be transferred from their original context of 
'work' to the context of 'travel', where selected aspects of travel are regarded as the new work.

\section{Conclusion}

This research clearly identifies dissatisfaction with non-self-imposed restrictions relating to work structures and behaviour as the underlying motivation for digital nomads on the second and third level (see Stormann, 1989). If combined with a perceived alienation from the original home environment, commonly based upon expectations, values and opportunities, professional freedom allows for spatial freedom through often international travel, in turn contributing to personal freedom by creating opportunities for self-development and learning. This is to create a holistic lifestyle that addresses individual needs and preferences in all areas of life as proposed by Parker (1971) and illustrated by Bowers (2011). Although the role of international travel is predominant in media-based information, it has been shown that it is not a necessary requirement for being a digital nomad, as location mobility can also be utilized on a more restricted scale. Digital nomads can thus be classified on three different levels (not including Level 0 - basic requirements) depending on the extent of their location mobility, beginning with those who are simply flexible in terms of their working place without incorporating travel. Second level digital nomads then retain their permanent residence but use the opportunity to travel more extensively, while third level digital nomads choose to abandon a permanent residence and fully commit to a life of mobility.

For the second and third level digital nomads participating in this research, professional and personal freedom - the traditional realms of work and leisure - are inextricably connected, as one provides the means for, is impacted by, and created based upon the other and vice versa. Prior to adapting the digital nomad lifestyle, participants conducted negatively perceived tasks predominantly in their working environment, however their lived experience shows that unfulfilling and extrinsically motivated activities could not be fully eliminated but were simply transferred from the realm of employment to the realm of travel. True leisure, as Stormann (1989) proposes, can thus indeed be achieved within work for the digital nomads represented in the sample, however this transfer comes at a cost that is to be paid in other areas of life. But as this trade-off is self-imposed and stems from the utilization of freedom, the overarching motive and goal of digital nomads, it appears as if the cost is paid more willingly. Results of this research suggest that the increasing development of and reliance on 
ICTs indeed has strong impacts on the interpretation of work and leisure in today's Western societies - common depictions often assume that work becomes predominant at the cost of leisure (Cropley \& Millward, 2009; Haworth \& Veal, 2004). Digital nomads show that this can also result in an opposite outcome where ICTs support the predominance of perceived leisure through their integration in a holistic lifestyle. This supports the argument that conceptualizations of work and leisure are required to fully move away from the dichotomy of employment time versus free time and must continue to focus on experiential and perceptional approaches when deepening our understanding of what is what. It is thus not sufficient to, as proposed by Roberts (2011), only reevaluate what leisure is; it is also necessary to reappraise our understanding of work. Whether the terms work and leisure are indeed the most appropriate expressions to understand how individuals structure their lives requires further attention.

Several limitations apply to this research. Sampling being based upon self-identification without further selective criteria led to digital nomads of the first definition level not being included but only appealed to those who self-identified with the terminology to the extent that they sought out online-based communities. The experiences of those who abandoned this lifestyle or are currently dissatisfied with it also require further attention to explore not only the positive and enriching developments but also the drawbacks of digital nomadism. In combination with a qualitative approach defined by relatively small sample sizes, it is to be expected that further distinctions can be made within the levels illustrated in Table 2. The sample (see Table 1) consists predominantly of individuals from Western cultures, thus excluding other potentially different cultural perspectives. Furthermore, due to the exploratory nature of this research, several aspects could not be touched upon. These include long-term developments, financial aspects and expenditure, potential differences based upon demographic characteristics and prior travel and work history, and also the psychological, socio-psychological and social factors and impacts of this lifestyle. In addition, which specific aspects factor into activities being perceived in a certain way could not be explored within this research and requires further attention to better understand how the original concepts of work and leisure can be determined, defined and interpreted within changing circumstances.

The increasing popularity of the digital nomad phenomenon illustrates that impacts of ICTs on travel can be comprehensive to the extent that they create new types of travellers. Discussing the type and form of travel in the digital nomad context went beyond the scope of 
this paper; yet is necessary to evaluate the potential of digital nomads for the tourism industry. Nevertheless, it has been shown that this new manifestation may constitute a new form of 'tourism' that, due to its often non-existent temporal limitations and the frequent lack of a home environment that is returned to, goes beyond common definitions of tourism and tourists. Further research is thus required to examine the travel aspect of digital nomads in more detail and to evaluate their contribution to research on tourism mobilities. In addition, it is uncertain if the travel undertaken by digital nomads falls into the category of recreational or business travel, or if such a distinction can indeed be made. Business travel comprises "all trips whose purpose is linked with the traveller's employment and business interests" (Davidson \& Cope, 2003, p. 3), adhering to the participants' focus on enhancing professional competences through travel. However, for most travel is not strictly necessary to perform work-related tasks and emerged as having more recreational qualities. Here, it must be considered that not all aspects are considered positive, beneficial and leisurely, providing potential for both new research avenues and enterprises on a highly relevant and current development. 


\section{References}

Ali, A., \& Frew, A. J. (2014). ICT and sustainable tourism development: an innovative perspective. Journal of Hospitality and Tourism Technology, 5(1), 2-16.

Altringer, B. (2015, 22.12.2015). Globetrotting Digital Nomads: The Future Of Work Or Too Good To Be True? Retrieved from http://www.forbes.com/sites/forbesleadershipforum/2015/12/22/globetrotting-digitalnomads-the-future-of-work-or-too-good-to-be-true/\#c3cc1c6523d4

Angen, M. J. (2000). Evaluating Interpretive Inquiry: Reviewing the Validity Debate and Opening the Dialogue. Qualitative Health Research, 10(3), 378-395.

Beatty, J. E., \& Torbert, W. R. (2003). The False Duality of Work and Leisure. Journal of Management Inquiry, 12(3), 239-252.

Bennion, N. (2013). What is a digital nomad? Retrieved from http://wanderingdesk.com/what-is-a-digital-nomad/

Berne, C., Garcia-Gonzalez, M., \& Mugica, J. (2012). How ICT shifts the power balance of tourism distribution channels. Tourism Management, 33(1), 205-214.

Best, S. (2010). Leisure Studies. Themes and Perspectives. London: SAGE Publications.

Biesalski, C. (2016). Von Reiseexzessen bis zur Homebase - Die Phasen des digitalen Nomadenlebens. Retrieved from http://www.planetbackpack.de/phasen/

Biesalski, C. (n.d.). About. Retrieved from http://www.connibiesalski.com/about/

Blanchflower, D. G. (2004). Self-employment: More may not be better. Swedish Economic Policy Review, 11(1), 15-73.

Blanda, S. (2016). Lauren Hom: From 9 to 5 to Digital Nomad. Retrieved from http://99u.com/articles/54017/lauren-hom-from-9-to-5-to-digital-nomad

Bookchin, M. (1982). The Ecology of Freedom. Palo Alto: Cheshire Books.

Bowers, T. (2011). Cultivating a Leisurely Life in a Culture of Crowded Time: Rethinking the Work/Leisure Dichotomy. World Leisure Journal, 49(1), 30-43.

Breedveld, K. (1996). Post-Fordist Leisure and Work. Loisir et Société / Society and Leisure, 19(1), 67-90.

Buhalis, D., \& O'Connor, P. (2005). Information Communication Technology Revolutionizing Tourism. Tourism Recreation Research, 30(3), 7-16.

Buttner, E. H., \& Moore, D. P. (1997). Women's Organizational Exodus to Entrepreneurship: Self-Reported Motivations and Correlates with Success. Journal of Small Business Management, 35(1), 34-46. 
Cropley, M., \& Millward, L. J. (2009). How do individuals 'switch-off' from work during leisure? A qualitative description of the unwinding process in high and low ruminators. Leisure Studies, 28(3), 333-347.

Csikszentmihalyi, M. (1991). Flow: The Psychology of Optimal Experience. New York: Harper Perennial.

Dal Fiore, F., Mokhtarian, P. L., Salomon, I., \& Singer, M. E. (2014). “Nomads at last'? A set of perspectives on how mobile technology may affect travel. Journal of Transport Geography, 41, 97-106.

David, E. (2014). Digital nomad case study. Retrieved from http://becomenomad.com/digital-nomad-case-study/

Davidson, R., \& Cope, B. (2003). Business Travel. Harlow: Pearson Education Limited.

Hamilton-Smith, E. (2006). Work, leisure and optimal experience. Leisure Studies, 11(3), 243-256.

Hamilton, C., \& Mail, E. (2003). Downshifting in Australia: A sea-change in the pursuit ${ }^{\wedge}$ happiness Canberra: The Australia Institute.

Hart, A. (2015). Living and working in paradise: the rise of the 'digital nomad'. Retrieved from http://www.telegraph.co.uk/news/features/11597145/Living-and-working-inparadise-the-rise-of-the-digital-nomad.html

Haworth, J., \& Lewis, S. (2005). Work, leisure and well-being. British Journal of Guidance \& Counselling, 33(1), 67-79.

Haworth, J., \& Veal, A. J. (2004). Introduction. In J. T. Haworth \& A. J. Veal (Eds.), Work and Leisure (pp. 1-11). London: Routledge.

Hilbrecht, M. (2007). Changing Perspectives on the Work-Leisure Relationship. Annals of Leisure Research, 10(3-4), 368-390.

Hsieh, H. F., \& Shannon, S. E. (2005). Three approaches to qualitative content analysis. Qualitative Health Research, 15(9), 1277-1288.

$\begin{array}{lllll}\text { Investopedia. (2017). Digital Nomad. } & \text { Retrieved from }\end{array}$ http://www.investopedia.com/terms/d/digital-nomad.asp

Kelly, K. (1998). New rules for the new economy: 10 radical strategies for a connected world. New York: Viking.

Kleiber, D. A. (2009). Fate control and leisure attitudes. Leisure Sciences, 2(3-4), 239-248.

Lamarque, H. (2015, 02.06.2015). The Rise of the Digital Nomad. Retrieved from http://www.huffingtonpost.com/hannah-lamarque/the-rise-of-the-digital$\underline{\text { n_b_7492482.html }}$ 
Lewis, S. (2003). The integration of paid work and the rest of life. Is Post-industrial work the new leisure? Leisure Studies, 22(4), 343-355.

Li, X., \& Hitt, L. M. (2008). Self-Selection and Information Role of Online Product Reviews. Information Systems Research, 19(4), 456-474.

Lincoln, Y. S., Lynham, S. A., \& Guba, E. G. (2011). Paradigmatic Controversies, Contradictions, and Emerging Confluences, Revisited. In N. K. Denzin \& Y. S. Lincoln (Eds.), The SAGE Handbook of Qualitative Research (pp. 97-128). Los Angeles: SAGE.

Lobo, F. (2006). The Work-Leisure Paradigm: The Stresses and Strains of Maintaining a Balanced Lifestyle. World Leisure Journal, 48(3), 22-32.

Makimoto, T., \& Manners, D. (1997). Digital Nomad. Chichester: Wiley.

Mokhtarian, P. L., Salomon, I., \& Handy, S. L. (2006). The impacts of ICT on leisure activities and travel: A conceptual exploration. Transportation, 33(3), 263-289.

Moore, K., Cushman, G., \& Simmons, D. (1995). Behavioral conceptualization of tourism and leisure. Annals of Tourism Research, 22(1), 67-85.

Parker, S. (1971). The future of work and leisure. New York: Praeger.

Patton, M. Q. (2015). Qualitative research \& evaluation methods: Integrating theory and practice (4th ed.). Thousand Oaks: SAGE Publications.

Pernecky, T. (2012). Constructionism. Critical Pointers for Tourism Studies. Annals of Tourism Research, 39(2), 1116-1137.

Primeau, L. A. (1996). Work and Leisure: Transcending the Dichotomy. The American Journal of Occupational Therapy, 50(7), 569-577.

Reichenberger, I., \& Schäfer, M. (2016). Sociology of Leisure. In H. Siller \& A. Zehrer (Eds.), Entrepreneurship und Tourismus (2 ed., pp. 243-249). Wien: Linde Verlag.

Reid, D. G., \& Mannell, R. C. (1994). The Globalization of the Economy and Potential New Roles for Work and Leisure. Loisir et Société / Society and Leisure, 17(1), 251-266.

Roberts, K. (2011). Leisure: the importance of being inconsequential. Leisure Studies, 30(1), 5-20.

Robinson, J., \& Godbey, G. (1997). Time for life: The surprising ways Americans use their time. . University Park: Pennsylvania State University Press.

Russell, J. (2013). 7 'digital nomads' explain how they live, work and travel anywhere in the world. Retrieved from https://thenextweb.com/insider/2013/12/15/7-digital-nomadsexplain-how-they-live-work-and-travel-anywhere-in-the-world/9/\#.tnw_cCiDaPL0

Seawright, J. (2016). The Case for Selecting Cases That Are Deviant or Extreme on the Independent Variable. Sociological Methods \& Research, 45(3), 493-525. 
Shaw, S. M. (2009). The meaning of leisure in everyday life. Leisure Sciences, 7(1), 1-24.

Small, J. (1999). Memory-work: a method for researching women's tourist experiences. Tourism Management, 20(1), 25-35.

Snedden, M. (2013, 30.08.2013). When work is a nonstop vacation. Retrieved from http://www.bbc.com/capital/story/20130829-when-work-is-a-nonstop-vacation

Spinks, R. (2015, 16.06.2015). Meet the 'digital nomads' who travel the world in search of fast Wi-Fi. Retrieved from http://www.theguardian.com/cities/2015/jun/16/digitalnomads-travel-world-search-fast-wi-fi

Stormann, W. (1989). Work: true leisure's home? Leisure Studies, 8(1), 25-33. doi:10.1080/02614368900390031

Surber, M. (1983). Work and Leisure: The Problem of Identity among Professional Workers. Loisir et Société / Society and Leisure, 6(2), 429-456.

Walsh, S. (2012). Travel and Earn Money Online - "The Experiment". Retrieved from http://discoveringice.com/lifestyle-design/post/the-experiment-lifestyle-design-travelwork-and-earn-money.html

Werthner, H., \& Klein, S. (1999). ICT and the Changing Landscape of Global Tourism Distribution. Electronic Markets, 9(4), 256-262.

Wikipedia. (2016). Digital Nomads. $\quad$ Retrieved from https://en.wikipedia.org/wiki/Digital_nomad 
Table 1. Sample Characteristics

\begin{tabular}{lllll}
\hline Gender & Age & Education & Profession & Nationality \\
\hline Female & 19 & High School & Freelancer (photography) & UK \\
\hline Male & 23 & Bachelor & Freelancer (copywriting, translation) & USA \\
\hline Female & 24 & Master & Entrepreneur (blogging, social media) & Germany \\
\hline Female & 25 & Master & Entrepreneur (photography, film) & Ireland \\
\hline Female & 25 & Bachelor & Entrepreneur (web design) & USA \\
\hline Female & 26 & Master & Freelancer (graphic design) & Sweden \\
\hline Female & 26 & Master & Entrepreneur (web, graphic design) & Norway \\
\hline Female & 27 & Bachelor & Freelancer (marketing) & Ireland \\
\hline Male & 27 & Bachelor & Entrepreneur (app developer) & UK \\
\hline Female & 28 & Master & Freelancer/Entrepreneur (programmer) & USA \\
\hline Male & 28 & Master & Freelancer (journalist, writer) & Ireland \\
\hline Male & 28 & Bachelor & Entrepreneur (travel agency) & Greece \\
\hline Female & 30 & Master & Remote Employee (programmer) & Canada \\
\hline Male & 31 & Master & Entrepreneur (travel blog) & Netherlands \\
\hline Male & 31 & Master & Freelancer (journalist) & Argentina \\
\hline Female & 32 & Bachelor & Freelancer/Entrepreneur (web design) & Scotland \\
\hline Female & 33 & Master & Entrepreneur (blogging, consultancy) & Germany \\
\hline Female & 33 & Master & Entrepreneur (sports, health) & USA \\
\hline Female & 35 & Master & Entrepreneur (marketing) & Germany \\
\hline Male & 44 & Master & Freelancer (journalist) & Germany \\
\hline Female & 45 & Master & Freelancer (marketing) & Brazil \\
\hline Male & 51 & Master & Freelancer (photography) & \\
\hline & & & & Baly \\
\hline
\end{tabular}


Table 2. Definition

\begin{tabular}{ll}
\hline Level & Definition \\
\hline $\mathbf{0}$ & Digital nomads are individuals who achieve location independence by \\
& conducting their work in an online environment, \\
\hline $\mathbf{1}$ & transferring this independence to mobility by not consistently working in \\
& one designated personal office space \\
\hline $\mathbf{2}$ & but using the possibility to simultaneously work and travel \\
\hline
\end{tabular}


Figure 1. Digital Nomad Holism

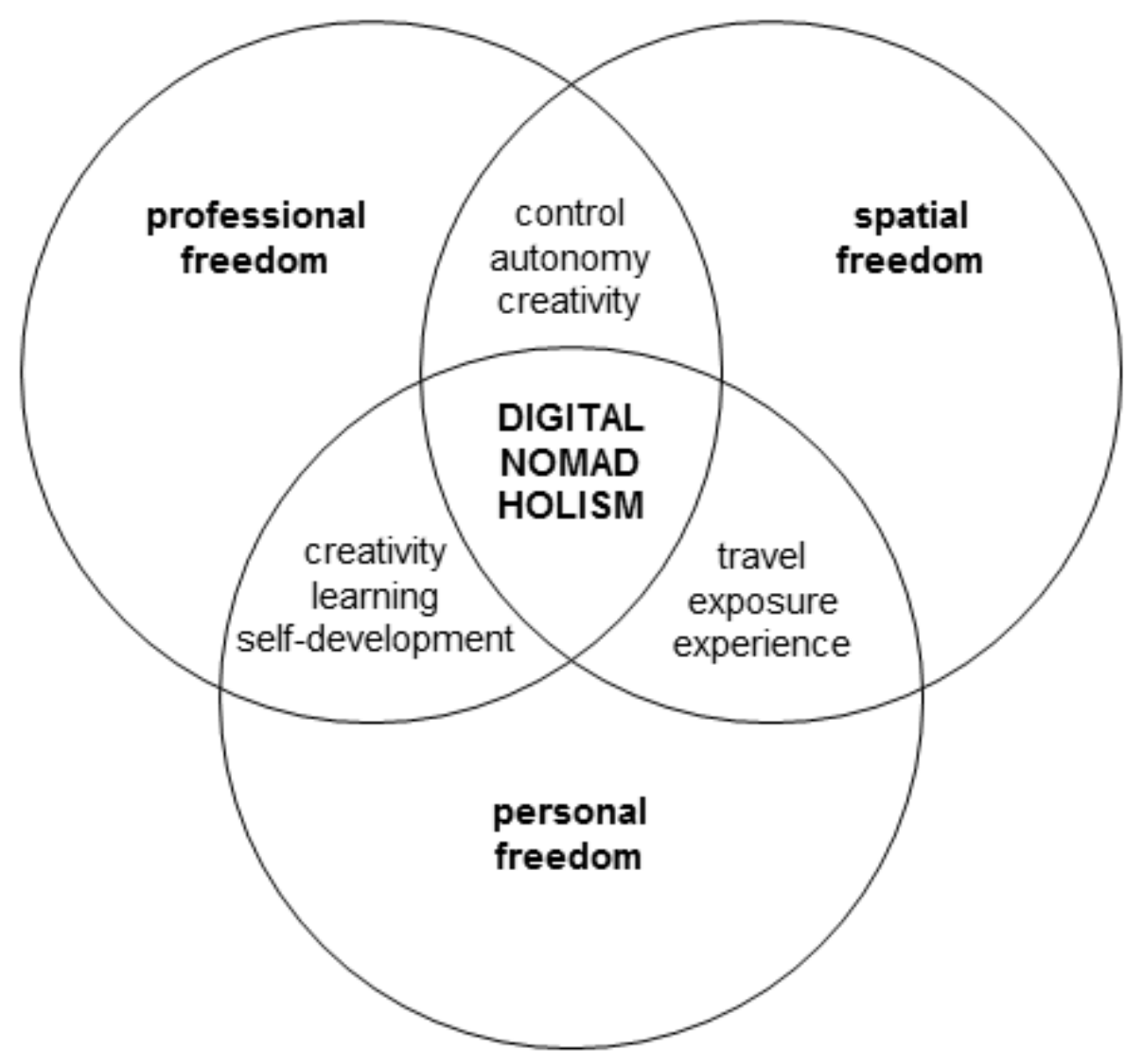

